\title{
Digital tablets to improve quality of life of COVID-19 older inpatients during lockdown
}

\author{
Radjiv Goulabchand ${ }^{1,2}(1) \cdot$ Helene Boclé $e^{3,4} \cdot$ Renaud Vignet $^{5} \cdot$ Albert Sotto $^{3,4} \cdot$ Paul Loubet $^{3,4}$
}

Received: 19 May 2020 / Accepted: 7 June 2020 / Published online: 13 June 2020

(c) European Geriatric Medicine Society 2020

Keywords Digital tablets $\cdot$ End-of-life $\cdot$ COVID-19

We are facing a historic pandemic, undermining both our health systems and societies. Fragile and geriatric patients' management represent a crucial challenge. They are particularly emotionally affected by Covid-19 because they are aware of its severe expected prognosis. Moreover, the benefit/risk ratio is often against their admission into intensive care units, leading to an optimised management within the medical departments. These situations lead to an anxiety state, worsening the dyspnea sensation. Furthermore, because a significant number also present with mild to severe cognitive impairment, the risk of developing spatiotemporal disorientation and delirium is greatly increased. Usually, caregivers can alleviate these stressful conditions by involving patients' families in their management: the presence of close relatives often helps calm patients and ease discomfort, especially during end-of-life situations.

However, during the current pandemic period, patients are alone in our hospitals: (1) all visits are forbidden to prevent risk of contagion; (2) lockdown and containment measures limit patients' relatives' movements; (3) relative's own fear of the disease overrides their wish to visit their relative, even in palliative cases. The negative impact of these factors on quality of life of our fragile patients prompted us to find

Radjiv Goulabchand

radjiv.goulabchand@chu-nimes.fr

1 Internal Medicine Department, CHU Nîmes Univ Montpellier, Place du Pr Debre, 30029 Nîmes, France

2 Inserm U1183, Institute for Regenerative Medicine and Biotherapy, St Eloi Hospital, 80 avenue Augustin Fliche, Montpellier, France

3 Department of Infectious and Tropical Diseases, CHU Nîmes Univ Montpellier, Nîmes, France

4 Inserm U1047, University of Montpellier, Nîmes, France

5 Addictions Department, CHU Nîmes, Nîmes, France a way to decrease their anxiety level, and consequently ease their conditions of hospitalisation.

We thus introduced digital tablets into our dedicated COVID-19 departments, as described elsewhere [1, 2]. Upon admittance of a patient too old or ill to use its own smartphone, we proposed to contact close relatives via the tablet. All patients and family accepted this technology. This was a time-consuming procedure. The tablets had to be set up (installation of Skype ${ }^{\circledR}$ ), contact details of family members uploaded, and we had to dedicate time in our workday to organise and assist the patients in these meetings. However, it was sincerely meaningful for patients and families. Patients were then more likely to follow our instructions.

This procedure was also very helpful in dealing with endof-life situations. We were able to offer meetings between family members and patients when they were still able to communicate, before they were too out of breath, under high flow oxygen therapy, or unconscious.

We suggest that this strategy helps families cope during the mourning process. They could see and talk to their relative, grasp how fragile they were, and sometimes realised how the disease was affecting them. Sometimes, they also realised it was their last chance to say "goodbye". We recommend initiating the meeting as soon as possible after admission, when communication is still possible, before the potential introduction of palliative care treatments. Moreover, we recommend that families and patients should be informed about the potential challenging situation of sharing moments with one's relative through digital tablets without the opportunity to physically touch or reassure them. We also would recommend a follow-up phone call to the family members to reassure them and estimate their psychological state.

This management could be proposed in several medical departments caring for fragile or geriatric patients, suffering from isolation during the lockdown era. As this procedure is controlled by health teams, it could help to improve 
their quality of life, and in some cases, help in end-of-life situations.

Acknowledgements We wish to thank Sarah Kabani for reading and revising the English wording of this letter.

\section{Compliance with ethical standards}

Conflict of interest None of the authors declare any conflict of interest concerning this topic.

Ethical approval All procedures performed in studies involving human participants were in accordance with the ethical standards of the institutional and/or national research committee and with the 1964 Helsinki declaration and its later amendments or comparable ethical standards.

Informed consent For this type of study formal consent is not required.

\section{References}

1 Wong B. Doctor Collects 650 Tablets So COVID-19 Patients Can Say Their Final Goodbyes. Huffington Post [Internet]. 2020 Apr 20 [cited 2020 Apr 23]. https://www.huffpost.com/entry/docto r-tablets-covid-19-patients-goodbyes_1_5e988500c5b65eae70a0 ec0e

2 Martineau P. iPads Are Crucial Health Care Tools in Combating Covid-19. Wired [Internet]. 2020 Apr 8 [cited 2020 Apr 23]. https://www.wired.com/story/ipads-crucial-health-tools-comba ting-covid-19

Publisher's Note Springer Nature remains neutral with regard to jurisdictional claims in published maps and institutional affiliations. 